\title{
Monitoring the Nociception Level Intraoperatively - An Initial Experiences
}

\author{
Pether Jildenstål1,2*, Katarina Hallén², Katarina Almskog ${ }^{3}$, Johan Sand ${ }^{3}$ and Margareta Warren Stomberg1 \\ ${ }^{1}$ Institute of Health and Care Sciences, University of Gothenburg, Sweden
}

${ }^{2}$ Department of Anesthesiology, Surgery and Intensive Care, Sahlgrenska University Hospital, Sweden

${ }^{3}$ Department of Anesthesiology and Intensive care, Queen Silvia Children's Hospital, Sweden

Submission: July 5, 2018; Published: July 25, 2018

*Corresponding author: Pether Jildenstål, Institute of Health and Care Sciences, University of Gothenburg, Sweden, Email: pether.jildenstal@gu.se

\section{Abstract}

Background: Estimating pain stimuli in the anesthetized patient can be difficult when based solely upon physiological parameters, especially when vasopressors are used as well. There is an increasing interest during general anesthesia to understand how optimal anesthesia changes by the level of noxious stimulation. Objectively, noxious stimulation measurement monitoring techniques are gaining interest. Although currently, its exact use in routine clinical practice is still not well proven. The aim of this study was to identify relationships between PMD 200 monitoring, Nociception Level (NOL-index) and monitored known physiological signs as well as outcomes during general anesthesia.

Method: Eight patients between the ages of 43 and 83 years old and scheduled for major head and neck surgery under general anesthesia were observed in this study. NoL index sensor was placed on one of the patient's fingers before anesthesia was induced, and values were extracted during the intraoperative period.

Results: NoL index values increased intraoperatively during nociceptive stimuli such as jaw lift, endotracheal intubation, catheterization of the bladder, and with surgical skin incision. NoL index increased at several occasions faster, and were more prominent than physiological parameters (BP, HR).

Conclusion: There are identified relationships between PMD 200 monitoring, NOL-index and nociceptive stimulation. NoL index tends to indicate nociceptive responses earlier and more often than hemodynamic outcomes. NoL index can be a physiological marker for optimal analgesic administration and an interesting complement to monitoring equipment intraoperatively worth further studies.

Keywords: NoL index; Pain; Pain estimation; Opioids; Anesthesia nurse; Vasoactive drugs

\section{Introduction}

There is an increasing interest to understand more how and when the depth of anaesthesia changes by the level of noxious stimulation during general anaesthesia. Nociception is the mechanism by which the central and peripheral nervous systems process information about noxious stimuli, whereas pain is the perception generated when this information reaches the cerebral cortex [1]. Under general anaesthesia, patients are unconscious, but their body still shows reflex responses to the surgical procedure, including changes in heart rate and blood pressure, eyes tearing or sweating [2].

During the past two decades, we have seen great advances in our understanding of pain. In particular, the molecular biology of pain and the way in which the central nervous system perceives and processes pain have been transformed [2]. The key to adequate pain management during general anaesthesia, is to assess its presence and severity, to identify those signs which require intervention, and by appreciating treatment efficacy [2,3]. In a recent review article (2015) by Cowen et al. [4], they addressed the latest important developments in the field regarding objective nociceptive measurements [4]. Non-invasive techniques utilized to measure pain have become clinically feasible [5]. There are several techniques in use, although none of them have been commercially successful globally. The PMD 200 nociception level index (NoL index ${ }^{\mathrm{TM}}$ ) is today a commercially available technique which is gaining growing interest [5]. The PMD 200 asses nociception stimuli by a multi-parametric algorithm [6]. The technology objectively quantifies the nociception stimuli (NoL index (0-100) and interpret the analgesic effect [6].

Due to the multifaceted nature of pain, PMD 200 focuses on the autonomic physiological integrated response to pain, rather than individual pain pathways. The device consists of a noninvasive finger probe which continuously records multiple pain 
related physiological signals - using four sensors namely a photo plethysmograph, galvanic skin response, as well as temperature and accelerometry [3]. In our study, we have in a clinical setting tested NoL index technique on patients during surgery under general anesthesia. The aim of this study was to identify relationships between PMD 200 monitoring, Nociception Level (NoL-index) and monitored known physiological signs as well as outcomes during general anesthesia.

\section{Materials and methods}

\section{Ethical considerations}

This article is based on a Master's thesis and ethical positions taken in the framework of a student thesis at Sahlgrenska Academy, University of Gothenburg. The study met the ethical requirements by the Helsinki Declaration [7].

\section{Patients and anesthesia}

This prospective observational study included 7 patients. Of the 8 patients assessed to be eligible for the study, one was excluded due to protocol violations. Inclusion criteria were according to the American Society of Anesthesiologists (ASA) risk classification I-IV, adults older than 18 yrs of age undergoing elective head and neck surgery under general anesthesia. Exclusion criteria included known neurological disorders, pregnancy, serve diabetes mellitus, uncontrolled hypertension, and drug addiction. All study patient received general anesthesia as per our department routine: Induction with intravenous Propofol 1-3mg/kg, Fentanyl 1.5$3 \mu \mathrm{g} / \mathrm{kg}$ and Rocuronium $0.6 \mathrm{mg} / \mathrm{kg}$. Maintenance of anesthesia was achieved with volatile anesthetic desflurane, supplemented with Fentanyl $1-1.5 \mu \mathrm{g} / \mathrm{kg}$ boluses. Muscle relaxation was maintained with Rocuronium when required. Vasopressors were administrated to $n=3 / 7$, (group 2). Group 1, $n=4$ did not receive vasopressors. All patients received standard monitoring including pulse oximetry, non-invasive blood pressure, 5-lead ECG, NMT and BIS. NoL index sensor was placed on one of the patient's fingers before anesthesia was initiated, and values were extracted during the intraoperative period, named NoL index.

\section{Data collection}

All data collection was conducted between 2018-04-10 2018-04-25 during seven surgical interventions at a university hospital in Sweden. Validation of the predetermined events in the observation schedule was performed in advance to resolve the facility, and the relevance of the moments but also if any additional data were required to be incorporated into the observation scheme. No changes needed to be made. The predetermined events were, jaw lift, endotracheal intubation, catheter insertion into the urethra, and skin incision. Also, physiological parameters (HR, BP, 02saturation, end- tidal carbon oxide and body temperature) were recorded every 1 or 5 minutes. Furthermore, Bispectral Index (BIS), Neuromuscular Monitoring (NMT), Nociception Level Index (NOL) and intraoperative drugs (opioids, hypnotics, vasopressor were also noted.
The observations were started immediately prior to the induction of anesthesia and ended when the surgical incision was dressed with sterile surgical dressing. The observation was made by the Master student who was not involved in the anesthesia process. Throughout the observation, the PMD 200 monitor's screen was turned away from the anesthesia staff during the length of the anesthetic. All anesthesia staff were informed of what was being observed during the anesthesia and for what purpose.

\section{Calculations and statistics}

Statistical calculation and analysis was performed by using IBM SPSS Statistics 24.0. Descriptive statistics were performed with Microsoft Excel 14.2 (CMicrosoft corp. 2010). Data is presented as, mean (SD), median (minimum-maximum) and numbers.

\section{Results}

Hence, 7 patients were included and observed in the observational study. The patients were comparable in respect of age, weight, ASA class, but not in gender (Table 1). Durations of anesthesia and surgery were almost similar, including the extent of surgical trauma in the group (Table 1,2). The NoL index derived from the PMD 200 showed increased values and were in relation to clinical status of blood pressure and heart rate (Figure 1-4). Although, NoL index increased at several occasions faster and, were more prominent than physiological parameters, (Table 3, Figure 1-4). The values increased significant (between 20-50 index values) from baseline through the predetermined pain stimuli and, ended spontaneously or were treated by opioids. With this study, patients who had a need for none/less (group 1) or increased infusion of vasopressors (group 2) during the anesthesia, PMD 200 showed episodes with increased NoL index and were not necessarily correlated to hemodynamics events. However, patients who did not receive any vasopressors showed often increased values even than the patient did not have physiological responses, (Table 3, Figure 1-4).

Table 1: Demographics.

\begin{tabular}{|c|c|}
\hline Patient characteristic data & $\mathbf{n = 7}$ \\
\hline Age, yrs & $63(43-83)$ \\
\hline Weight $(\mathrm{kg})$ & $82(50-103)$ \\
\hline Anesthesia, minutes & $186(120-290)$ \\
\hline Gender M/F & $6 / 1^{*}$ \\
\hline ASA class $2-3$ & $1 / 6^{*}$ \\
\hline
\end{tabular}

Median value, average (min-max) and *numbers

Table 2: Type of surgical procedure.

\begin{tabular}{|c|c|}
\hline Type of surgery & $\mathbf{n = 7}$ \\
\hline Mandible surgery & 3 \\
\hline Major neck surgery & 4 \\
\hline
\end{tabular}


Table 3: Group comparison, Mean values of HR, SBP, NoL index $>25$.

\begin{tabular}{|c|c|c|}
\hline & $\begin{array}{c}\text { Group 1 } \\
\text { Pat 2, 3, 6 \& 8 }\end{array}$ & $\begin{array}{c}\text { Group 2 } \\
\text { Pat 4, 5 \& 7 }\end{array}$ \\
\hline HR mean, \pm SD & $71.8 \pm 11$ & $72.8 \pm 7.4$ \\
\hline Sbp mean, \pm SD & $102 \pm 3.36$ & $118 \pm 7.9$ \\
\hline NOL INDEX mean, \pm SD & $14.5 \pm 4$ & $14.6 \pm 5.6$ \\
\hline NOL INDEX >25 (n) & 134 & 116 \\
\hline $\begin{array}{c}\text { NOL INDEX >25 + not affected } \\
\text { hemodynamics* (n) }\end{array}$ & $58 / 134$ & $54 / 116$ \\
\hline
\end{tabular}

${ }^{*} 10 \%$ increase from mean in heartrate and/or blood pressure at time of reading

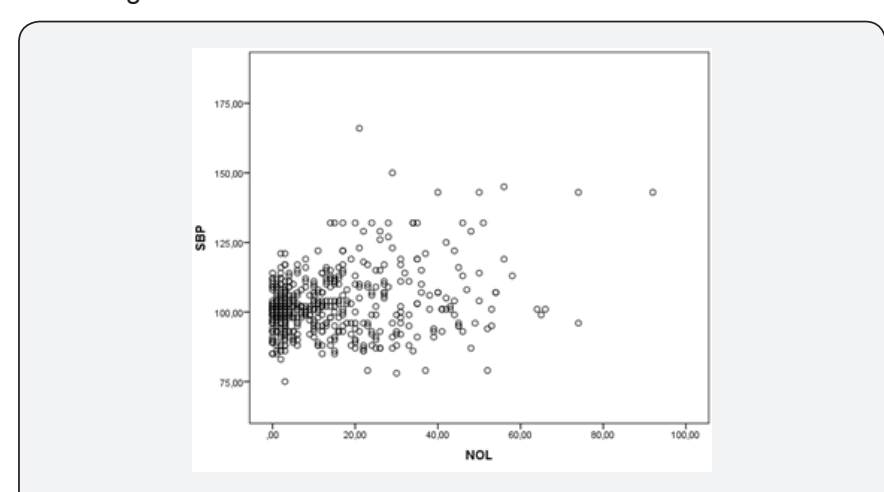

Figure 1: Scatterplot of SBP/NoL index, group 1"less vasoactive agents" (patient 4,5,7 excluded).

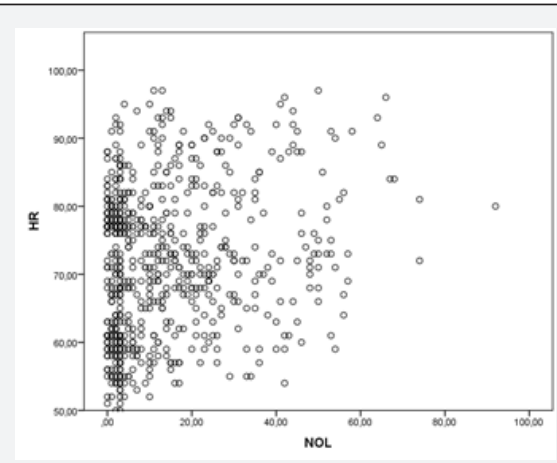

Figure 2: Scatterplot of HR/NoL index, group 1, (less vasoactive drugs), (patient 4,5,7 excluded).

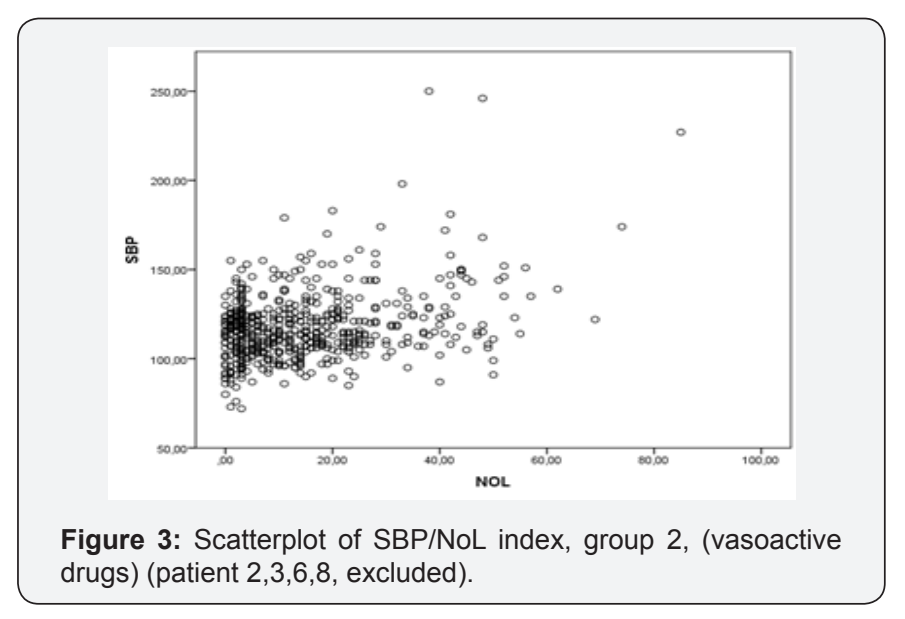

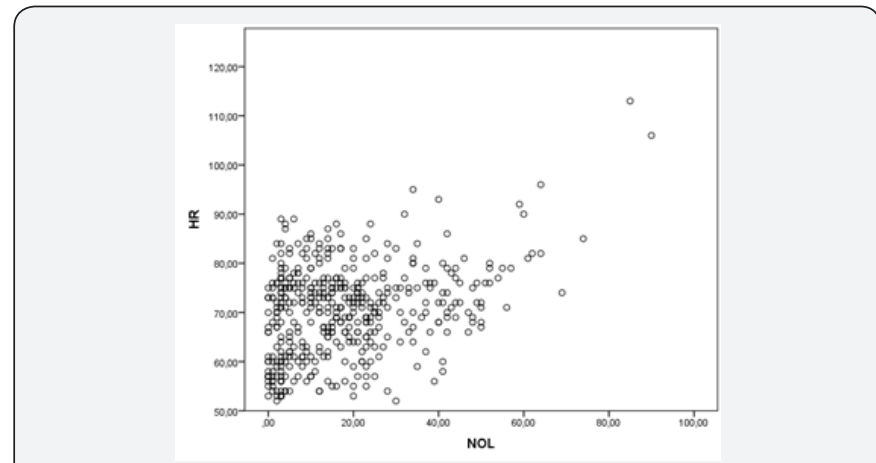

Figure 4: Scatterplot of $\mathrm{HR} / \mathrm{NoL}$ index, group 2, (vasoactive drugs) (patient 2,3,6,8, excluded). (In group 2, patient number 4 HR excluded due to pacemaker).

The mean of NoL index in patients prior to anesthesia was 20 (baseline). Listed NoL index before a painful stimulation induced or pain relief was given varied between values of 2-35. Nine events in total were identified, including the four predetermined events: (jaw hip, endotracheal intubation, catheterization of the urinary bladder (CAD), skin incision, number of opioid administrations, hypnosis and BIS intraoperative, hemodynamic correlation, and body temperature). After anesthetic induction, when jaw lifts and intubation were performed, NoL index increased in all study patients. The mean of NoL index at jaw lifts was 45 and at intubation 52. At two out of seven intubations, NoL index rose to a high level, up to 90 and 92 respectively. In all seven cases blood pressure increased during these events. The mean of NoL index was 44 at urinary bladder catheter insertion.

All patients received Fentanyl as analgesic of choice 5-15 minutes prior to surgical incision, although in five out of seven cases NoL index increased anyways. The average of NoL index during skin incision was 34 . To some patient's, opioids were given when systolic blood pressure increased $10-15 \%$, although NoL index did not indicate pain stimulating events. In a couple of cases, we could detect a similar variation by increased BIS index and NoL index during the same time period.

\section{Discussion}

General anesthesia is characterized by hypnosis and painlessness. Painful stimuli during surgery cause nociceptive autonomic response that must be inhibited. Monitoring of the hypnotic effect is possible from different EEG variables [8] while monitoring of nociception during surgery is a need under construction as a routine [9]. Although measures to identify intraoperative pain responses exist, there is a need to develop and clinical test a new index with improved accuracy. We tested monitoring of NoL gathering numerical value from several single physiological variables giving an index of nociception level NoL and found this monitoring valuable and promising as a supplement to other monitoring equipment. Increase in the NoL index was correlated with jaw lifting, endotracheal intubation, CAD and skin incision, all known as painful stimuli during anesthesia. Similar, the increased NoL index was appropriately decreased by analgesic administration, in accordance to Edry et al. (5). 
The highest NoL index was found during intubation which is in accordance to Stockle et al. (10) who found similar. BIS value prior to intubation is inadequate to prevent an awareness reaction to intubation during propofol/alfentanil anesthesia why optimal analgesic must protect the risk of awareness during endotracheal intubation [11]. There was also some compliance between NoL and hemodynamic changes, such as pulse and blood pressure. Tissue damage caused by skin incision causes activation of the sympathetic nervous system, which gives increased heart rate and increase in arterial blood pressure. These hemodynamic changes can be an indication of a nociceptive response [12] and, at the same time generate increased NoL. As there is individual variability in the physiological response to vasopressor therapy in this study, the result should therefore be interpreted with caution [13].

Similar to American Society of Anesthesiologists [5] we found that an algorithmic combination to process multiple physiologic measures, provide a better measure of the autonomic reflex response to painful stimulation during surgery, compared to the traditional monitoring of individual factors such as changes in heart rate and blood pressure and constitutes a valuable complement to other intraoperative monitoring. Further value from NoL may be to identify painful moments during surgery and to facilitate optimal administration of analgesics. The main limitations in this study are the small number of observations included and unclearness of vasopressors impact on NoL.

\section{Conclusion}

There are identified relationships between PMD 200 monitoring, NOL-index and nociceptive stimulation. NoL index tends to indicate nociceptive responses earlier and more often than hemodynamic outcomes. NoL index can be a physiological marker for optimal analgesic administration and an interesting complement to monitoring equipment intraoperatively worth further studies.

\section{Acknowledgement}

We thank the RNAs students Tommy Ehrman and Andreas Axelsson for their work with the Master`s thesis.

\section{Conflict of Interest}

None of the authors have any conflict of interest. Funding, this research did not receive any specific grant from funding agencies in the public, commercial, or not-for-profit sectors.

\section{Authors' Contributions}

PJ has planned the study, prepared the first version of manuscript and MWS, KH, KA and JS has taken part in the result presentation and writing of manuscript. All authors were involved in manuscript and approved the final version. The RNA students TE and AA conducted the observations and performed a Master`s thesis from the data.

\section{References}

1. Heinricher MM, Tavares I, Leith JL, Lumb BM (2009) Descending control of nociception: Specificity, recruitment and plasticity. Brain research reviews 60(1): 214-225.

2. Colvin LA, Rowbotham DJ (2013) Managing pain: recent advances and new challenges. Br J Anaesth 111(1): 1-3.

3. Ben Israel N, Kliger M, Zuckerman G, Katz Y, Edry R (2013) Monitoring the nociception level: a multi-parameter approach. Journal of clinical monitoring and computing 27(6): 659-668.

4. Cowen R, Stasiowska MK, Laycock H, Bantel C (2015) Assessing pain objectively: the use of physiological markers. Anaesthesia 70(7): 828847.

5. Edry R, Recea V, Dikust Y, Sessler DI (2016) Preliminary Intraoperative Validation of the Nociception Level Index: A Noninvasive Nociception Monitor. Anesthesiology 125(1): 193-203.

6. NoL. Retrieved 2018-07-02 from https://www.medasense.com/NoL index-nociception-level-

7. 2013 World Medical Association Declaration of Helsinki: ethical principles for medical research involving human subjects. JAMA 310(20): 2191-2194

8. Marchant N, Sanders R, Sleigh J, Vanhaudenhuyse A, Bruno MA, et al. (2014) How electroencephalography serves the anesthesiologist. Clinical EEG and neuroscience 45(1): 22-32.

9. Kang H (2015) Intraoperative nociception monitoring. Anesthesia and Pain Medicine 10(4): 227-234.

10. Stockle PA, Julien M, Issa R, Decary E, Brulotte V, et al. (2018) Validation of the PMD100TM and its NOLTM Index to detect nociception at different infusion regimen of remifentanil in patients under general anaesthesia. Minerva Anestesiol.

11. Schneider G, Wagner K, Reeker W, Hanel F, Werner C, et al. (2002) Bispectral Index (BIS) may not predict awareness reaction to intubation in surgical patients. Journal of neurosurgical anesthesiology 14(1): 7-11.

12. Jänig W (2012) Autonomic reactions in pain. Pain 153(4): 733-735.

13. Bockenstedt TL, Baker SN, Weant KA, Mason MA (2012) Review of vasopressor therapy in the setting of vasodilatory shock. Advanced emergency nursing journal 34(1): 16-23. 
(CC) This work is licensed under Creative Commons Attribution 4.0 License DOI: 10.19080/JAICM.2018.07.555709

\section{Your next submission with Juniper Publishers} will reach you the below assets

- Quality Editorial service

- Swift Peer Review

- Reprints availability

- E-prints Service

- Manuscript Podcast for convenient understanding

- Global attainment for your research

- Manuscript accessibility in different formats

( Pdf, E-pub, Full Text, Audio)

- Unceasing customer service

Track the below URL for one-step submission https://juniperpublishers.com/online-submission.php 